\title{
Inflammation and Immunotherapy in Atherosclerosis
}

\author{
Konstantinos S. Mylonas ${ }^{1}$, Alkistis Kapelouzou ${ }^{2}$, Michael Spartalis ${ }^{3}$, \\ Dimitrios Schizas ${ }^{1}$, Eleftherios Spartalis ${ }^{4}$, Christos Bakoyiannis ${ }^{5}$, Dimitrios Iliopoulos ${ }^{6}$, \\ Theodoros Liakakos ${ }^{1}$, Nikolaos Nikiteas ${ }^{4}$
}

'First Department of Surgery, Laiko General Hospital, National and Kapodistrian University of Athens, Athens, Greece ${ }^{2}$ Clinical, Experimental Surgery \& Translational Research, Biomedical Research Foundation Academy of Athens, Athens, Greece

${ }^{3}$ Department of Cardiology, Onassis Cardiac Surgery Center, Athens, Greece

${ }^{4}$ Second Propaedeutic Department of Surgery, Laiko General Hospital, National and Kapodistrian University of Athens, Athens, Greece

${ }^{5}$ Division of Vascular Surgery, Laikon General Hospital, National and Kapodistrian University of Athens, Athens, Greece

${ }^{6}$ Fourth Department of Cardiac Surgery, HYGEIA Hospital, Athens, Greece

\begin{abstract}
Both the innate and adaptive arms of the immune system partake in various steps of atherogenesis. Recent studies have unraveled proatherogenic properties to clonal hematopoiesis of indeterminate potential (CHIP). Krüppel-like factors (KLFs) are transcription factors regulating pathways that confer atheroresistant and antiinflammatory effects. Pharmaceutical targeting of inflammatory cascades could be beneficial in battling atheromatosis. Statins, aspirin, and other anti-platelet medication have off-target immune-modulatory effects. Canakinumab, a monoclonal antibody targeting IL-1 $\beta$ significantly reduces major adverse cardiovascular events. Nevertheless, according to the CANTOS study this does come with an increased risk of sepsis. As shown in the LoDoCo and COLCOT trials, low-dose colchicine also exerts substantial cardiovascular protection without predisposing to infections. Targeting other pathways has yet to provide an evidence of cardiovascular benefit.

KEY WORDS: Atheromatosis, coronary artery disease, inflammation, pharmaceutical interventions, canakinumab, colchicine
\end{abstract}

\section{INTRODUCTION}

Atherosclerotic plaquesusuallydevelop at sites of hemodynamic shear stress, such as curvatures, branches,

\section{Corresponding author:}

Konstantinos S. Mylonas, MD

First Department of Surgery, Laiko General Hospital,

National and Kapodistrian University of Athens

AgiouThoma 17, Athens, 11527

Tel.: +306987024653

E-mail:ksmylonas@gmail.com and bifurcations of large arteries where disturbed blood flow prevails. ${ }^{1}{ }^{2}$ This is typically characterized by laminar flow separation, transient flow reversals, and shear forces that predispose to atherogenesis. On the other hand, pulsatile unidirectional laminar undisturbed flow usually prevails in atheroresistant regions. ${ }^{3}$

Of note, Rudolph Virchow (1821-1902) recognized inflammation in histological preparations of coronary 
arteries and hypothesized that inflammation may play a causal role in the development of atherosclerosis. Despite this landmark observation, drug development programs have primarily focused on cholesterol metabolism alone, and inflammation has received less attention over time. That said, during the past several decades extensive observations provided additional evidence supporting the importance of inflammation in the development and destabilization of atherosclerosis. ${ }^{4}$

In the present work, we summarize the pathophysiology of athero-inflammation and provide an up-to-date synopsis of various pharmaceutical interventions that may be useful in improving cardiovascular outcomes throughreducing the immunologic component of atherogenesis.

\section{EXPLORING THE IMPACT OF INFLAMMATION ON ATHEROMATOSIS}

\section{Macrophages and interleukins}

Traditional cardiovascular risk factors such as dyslipidemia, smoking, and hypertension induce endothelial damage. ${ }^{5}$ As a response to local tissue injury, the endothelium upregulates the transcriptional factor NF-kB and producesa variety of chemokines that favor leukocyte adhesion, such asendothelin, E-selectin, vascular and inter-cellular adhesion molecules including vascular cell adhesion molecule (VCAM-1)and intercellular Adhesion Molecule 1 (ICAM-1) (Figure 1). Rolling leukocytes adhere to the endothelium and penetrate beneath the endothelial layer to reach the subintimal space. Modified lipoproteins are recognized byindigenous dendritic cells and macrophages. ${ }^{6}$ Bone marrow derived monocytes subsequently approach the intima via chemotaxis. As they enter the subendothelial space, they differentiate into macrophages and engulf modified low-density lipoproteins (LDL), where excess cholesterol is esterified for storage in lipid droplets, giving macrophages their textbookfoam-like appearance.

Subsequently, the presence of cholesterol crystals leads to the upregulation of scavenger receptors (such as cluster differentiation 36 -CD36) which in turn induce downstream activation of the toll-like receptor pathway. Thenucleotide-binding domain (NOD)-like receptor protein 3 (NLRP3) inflammasome is ultimately activated in the cytoplasm of the macrophages. Pro-interleukin- $1 \mathrm{~b}$ (IL-1b) and IL-18 are cleaved by the inflammasomeand secreted as activated cytokines. ${ }^{8}$ Of note, IL-1 bis thought to be a very potent determinant of athero-inflammation. ${ }^{9}$ In the extracellular space, interleukins activate T-cells and induce the release of reactive oxygen species (ROS) andmetalloproteinases. ${ }^{10}$ Althoughcertain T-cells, such as T-helper-1 (Th1) cells, play a pro-atherogenic role, regulatory T-lymphocytes (TREGs) curtail atheromatosis via secreting TGF-beta and IL-10. T-helper 17 secrete IL-17 which also helps stabilize atheromatic plaques. ${ }^{11}$

\section{Smooth muscle cells (SMCs)}

Smooth muscle cells (SMCs) possess remarkable phenotypic plasticity that allows rapid adaptation to fluctuating environmental stimuli, including atherosclerosis drivers. Indeed, during the development of an atherosclerotic plaque, certain SMCs can transition from a primarily contractile, nonproliferating phenotype to a proliferating, migratory and matrix-secreting state that populates the arterial intima. Furthermore, such SMCs engulf modified lipids and adopt a "macrophage-like" phenotype, expressing macrophage markers on their surface and develop phagocytic activity. ${ }^{12}$
Types of cells

Endothelium
Molecular cascades

NF-kB

VCAM-1, ICAM-1, selectins eNOS

KLFs

Dendritic cells
Macrophages
Smooth-muscle
cells

CHIP

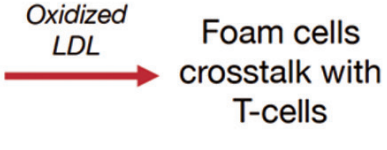
crosstalk with

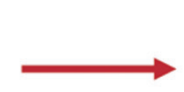
NLRP3 inflammasome
IL-1b, IL-18

KLFS

TET-2

FIGURE 1. Immuno-inflammatory mechanisms related to atherosclerosis. 


\section{Clonal hematopoiesis of indeterminate potential (CHIP)}

Clonal hematopoiesis of indeterminate potential (CHIP) is common among older individuals. CHIP is an expanded somatic blood-cell clone seen in people without other hematologic abnormalities. Preliminary data suggested an association between CHIP and atherosclerotic cardiovascular disease. ${ }^{13}$ A subsequent Harvard-led study, confirmed this observation demonstrating a two-fold risk incrementin myocardial infarction (MI) and stroke in CHIP-carriers. Additionally, there was a close association between premature atherosclerotic cardiovascular events and CHIP with a quadruple risk increase in patients younger than 50 years. ${ }^{14}$ Interestingly, certain groups have suggested that a low degree of chronic inflammation in patients with risk factors for atherosclerosis may promote somatic changes in hematopoietic cell lines and eventually CHIP. ${ }^{15,16}$ Among a large number of mutation-susceptible genes, Tet methylcytosine dioxygenase 2 (TET2) appears to be the most commonly affected one leading to CHIP. Indeed, TET2 mutations have been shown to promote clonal hematopoietic expansion and accelerate atherosclerosis in hyperlipidemic mice. ${ }^{17}$ In animal models, TET2 deficient macrophages induced increased expression of the NLRP3 inflammasome and IL-1b which as previously described favor atherogenesis. ${ }^{18}$

\section{Krüppel-like factors}

Endothelium in atherosusceptible regions is genetically different compared to that of atheroresistant sites. Krüppel-like factors(KLFs)are zinc-finger regulatory transcription factors regulating genetic pathways that confer atheroresistant, anti-inflammatory, and anti-thrombotic features to vascular endothelial cells and monocytes/ macrophages. ${ }^{19-21}$ In vivo, dysregulation of the following KLF pathways has been shown to promote atherogenesis. Recent data have shown that KLFs (especially KFL2 and KLF4) are crucial for shear stress- transcriptional activation of ITPR3. This novel mechanism contributes to the calcium-dependent eNOS (endothelial nitric oxide synthase) activation and endothelial homeostasis. ${ }^{22} \mathrm{KLF}$ overexpression induces several other anti-inflammatory and antithrombotic factors such and thrombomodulin, while decreasing TNFa-induced VCAM-1, E-selection, and tissue factor expression ${ }^{19}$. KLFs bind to the promoter of the VE-cadherin gene in mature ECs and inducesvascular endothelial (VE) -cadherin transcription. ${ }^{23} \mathrm{KLFs}$ also attach to three predicted KLF consensus binding sites in the Connexin40 (Cx40) promoter. Importantly, both VE-cadherin and Cx40-mediated gap junctional communication are known to contribute to a healthy endothelium by propagating anti-inflammatory signals between ECs. ${ }^{24}$

KLFs also protect against atheromatosis by regulating macrophage/monocyte polarization. Particularly, KLFs induce phenotypic shifts in monocytes from the pro-inflammatory M1 to the anti-inflammatory M2 type. Mechanistically, KLFs have been found to cooperate with STAT6 (signal transducer and activator of transcription 6) to promote quintessential M2 targets. On the other hand, inhibition of the M1 phenotype is achieved by inhibiting NF-kB transcriptional activity via sequestration of its critical coactivators such as p300 and P300/CBP-associated factor (PCAF). ${ }^{25}$

\section{Pharmaceutical targeting of atheromatosis- related inflammation}

Table 1 summarizes all beneficial anti-inflammatory therapies, the studies in which these therapies have been investigated, and their clinical effectiveness. Table 2 lists agents which failed to show any clinical benefit.

\section{Current off-target anti-inflammatory therapies}

\section{Statins}

Statins afford cardiovascular protection by reducing LDL levels through the inhibition of HMG-CoA (3-hydroxy3-methylglutaryl-Coenzyme A) reductase. That said, there is ample data suggesting that statins also decrease cardiovascular inflammation. First, statins inhibit prenylated

TABLE 1. Anti-inflammatory agents that provide significant cardiovascular risk reduction

\begin{tabular}{lccc}
\hline Target & Agent & Trial & Number of participants \\
\hline IL-1b & Canakinumab & CANTOS & 10,061 \\
NLRP3 inflammasome & Colchicine & LoDoCo & 532 \\
NLRP3 inflammasome & Colchicine & COLCOT & 4,745 \\
NLRP3 inflammasome & MCC950 & Encouraging results in preclinical models \\
\hline
\end{tabular}

Abbreviations: IL-1b: interleukin 1b, NLRP3: nucleotide-binding domain (NOD)-like receptor protein 3, CANTOS: Canakinumab Anti-Inflammatory Thrombosis Outcomes trial, LoDoCo: Low Dose Colchine trial, COLCOT: Colchicine Cardiovascular Outcomes trial 
TABLE 2. Anti-inflammatory agents that failed to provide significant cardiovascular risk reduction

\begin{tabular}{lccc}
\hline Target & Agent & Trial & Number of participants \\
\hline Oxidized LDL & Succinobulol & ARISE & 6144 \\
sPLA2 & Verespladib & VISTA-16 & 5000 \\
LPPLA2 & Darapladib & STABILITY & 15,000 \\
LpPLA2 & Darapladib & SOLID-TIMI 52 & 13,000 \\
P-selectin & Inclacumab & SELECT-ACS & 544 \\
P-selectin & Inclacumab & SELECT-CABG & 380 \\
IL-1RI & Anakinra & IL-HEART & 182 \\
5-LO & Atreleuton & NCTO0358826 & 191 \\
P38 MAPK & Losmapimod & LATITUDE-TIMI & 3,503 \\
Dihydrofolatereductaseinhibitor & Methotrexate & CIRT & 4,786 \\
\hline
\end{tabular}

Abbreviations: sPLA2: secretory phospholipase A2, LpPLA2: lipoprotein-associated phospholipase A2, interleukin 1RI: IL-1RI, interleukin 1A: IL-1A, 5-LO: 5-lipoxygenase, MAPK: mitogen-activated protein kinase, ARISE: Aggressive Reduction of Inflammation Stops Events trial, VISTA-16:Vascular Inflammation Suppression to Treat Acute Coronary Syndrome, STABILITY: STabilization of Atherosclerotic plaque By Initiation of darapLadlbTherapY trial, SOLID-TIMI 52: Stabilization Of pLaquesusIngDarapladib-Thrombolysis In Myocardial Infarction 52 trial, SELECT-ACS: P-Selectin Antagonist Inclacumab on Myocardial Damage After Percutaneous Coronary Intervention for Non-ST-Segment Elevation Myocardial Infarction trial, SELECT-CABG: Effects of P-Selectin Antagonist Inclacumab in Patients Undergoing Coronary Artery Bypass Graft Surgery trial, LATITUDE-TIMI: Losmapimod to Inhibit p38 MAP Kinase as a Therapeutic Target and Modify Outcomes After an Acute Coronary Syndrome trial, CIRT: Cardiovascular Inflammation Reduction Trial

protein production and the mevalonate pathway whileinducingKLF 2 and NOS expression. ${ }^{26}$ Statins also reduce endothelial cell activation and inhibit the induction of major histocompatibility complex Class II expression by interferon (IFN)-g thereby decreasing T-cell activation. ${ }^{27}$

\section{Aspirin and anti-platelet therapy}

Aspirin and other antiplatelet medication inhibit Pselectin which in turn reduces the release of inflammatory chemokines. Low-dose aspirin also triggers the synthesis of 15-epi-lipoxin A4, which mediates NOS synthesis and limits endothelial cell activation and leukocyte recruitment. ${ }^{28}$ In human aortic endothelial cell lines, intracytosolic NLRP-1 expression also is attenuated by aspirin, without direct platelet-endothelial cell interaction. ${ }^{29} \mathrm{~A}$ landmark study by Brigham and Women's Hospital, showed a 55\% reduction in the risk for myocardial infarction in healthy men with high CRP levels taking aspirin. ${ }^{30}$

Moreover, P2Y12 inhibitors reduce platelet release of pro-inflammatory a-granule contents and the formation of pro-inflammatory platelet-leukocyte aggregates. Clinical evidence shows that P2Y12 inhibition by clopidogrel is associated with a reduction in platelet-related mediators of inflammation, such as soluble P-selectin and CD40L. Compared to aspirin alone, the addition of clopidogrel, also significantly reduces markers of systemic inflammation such as TNF and CRP following ACS. The more potent thienopyridine $\mathrm{P} 2 \mathrm{Y} 12$ inhibitor, prasugrel, has been shown to decrease platelet P-selectin expression and platelet-leukocyte aggregate formation more extensively compared to clopidogrel. ${ }^{31}$ The PLATO study suggested that the novel P2Y12 inhibitor ticagrelor might improve clinical outcomes from pulmonary infections and sepsis compared to clopidogrel in patients with ACS. ${ }^{32}$ Ticagrelor is a more potent P2Y12 inhibitor than clopidogrel and also inhibits cellular adenosine uptake via equilibrative nucleoside transporter (ENT) 1, whereas clopidogrel does not. For all of the aforementioned reasons, aspirin and P2Y12 inhibitors seem to be extremely useful tools in reducing cardiovascular inflammation.

\section{Canakinumab}

The randomized, double-blind Canakinumab AntiInflammatory Thrombosis Outcomes (CANTOS) trial, investigated canakinumab, a monoclonal antibody targeting interleukin-1 $\beta .{ }^{33}$ The study involved 10,061 patients with prior myocardial infarction and a high-sensitivity C-reactive protein (hsCRP) level of $2 \mathrm{mg} / \mathrm{lt}$ or more. The patients were randomized to receive one of 3 canakinumab doses (50 $\mathrm{mg}, 150 \mathrm{mg}$, and $300 \mathrm{mg}$ ) administered subcutaneously every 3 months or placebo. All patients received standard of care therapy and serum LDL levels at enrollment had to be within guidelines dictated limits.

The primary efficacy endpoint of major adverse cardiovascular events (MACEs): nonfatal myocardial infarction, nonfatal stroke, or cardiovascular death was achievedin the $150 \mathrm{mg}$ study arm (hazard ratio [HR]0.85; $95 \% \mathrm{Cl}$, 0.74 to $0.98 ; p<0.05)$ dose. The secondary endpoint in- 
cluded a combination ofcardiovascular death, non-fatal myocardial infarction and stroke plus hospitalization for unstable angina leading to urgent revascularization and was again significantly lower with the $150 \mathrm{mg}$ regimen (HR 0.83; 95\% Cl, 0.73 to 0.95; $\mathrm{p}=0.005$ ). It should be emphasized that canakinumab was associated with a higher incidence of fatal infections than placebo $(0.31$ events $x 100$ person years for all combined doses vs. 0.18 events $x 100$ person years for placebo; $p=0.02)$. That said, canakinumab dosing did not influence all-cause or cardiovascular mortality.

The CANTOS trial group also performed prespecified subanalyses to identify which patient groups benefit the most from cananinumab and whether reductions in hsCRP levels correlate with clinical benefits. ${ }^{34}$ Compared to placebo, MACEswere significantly reduced in patients with an hsCRP level $<2 \mathrm{mg} / \mathrm{L}$ after 3 months of treatment (HR: 0.75 (95\% Cl 0.66-0.85; $\mathrm{p}<0.0001)$ but not in those with an $\mathrm{hsCRP}>2 \mathrm{mg} / \mathrm{L}$. Among patients withhsCRP $<2 \mathrm{mg} / \mathrm{L}$ there was also a $31 \%$ reduction in cardiovascular and all-cause mortality ( $p<0.0004$ and $p<0.0001$, respectively).

A subsequent analysis of CANTOS data, compared nearly 2000 patients with chronic kidney disease (CKD: estimated glomerular filtration rate $<60 \mathrm{ml} / \mathrm{min} / 1.73 \mathrm{~m} 2$ ) with the remaining approximately 8000 patients enrolled in CANTOS. ${ }^{35}$ Canakinumab reduced MACEs in CKD patients and was particularly effective in those who achieved a level of hsCRP $<2 \mathrm{mg} / \mathrm{L}$ after the first drug dose. In patients with $\mathrm{hsCRP}<2 \mathrm{mg} / \mathrm{L}$, cardiovascular and all-cause mortality were also significantly reduced(with no adverse laboratory or clinical renal events).

\section{Colchicine}

Colchicine is a cheap, per os medication, with potent anti-inflammatory properties that was initially extracted from the plant Colchicum autumnale ("meadow saffron"). Colchicine exerts substantial anti-inflammatory effects through the inhibition of tubulin polymerization and microtubule generation and affects cellular adhesion molecules such as selectins, inflammatory chemokines (i.e. leukocyte function-associated antigen-1), and the inflammasome. ${ }^{36,37}$ This medication has been traditionally used to treat acute gout attacks, familial Mediterranean feveras well as acute pericarditis. In the randomized-controlled LoDoCo (low dose colchicine) trial, $0.5 \mathrm{mg} /$ day colchicine was tested in over 500 patients with stable coronary artery disease (CAD).$^{38}$ The primary outcome of acute coronary syndrome, out-of-hospital cardiac arrest, or non-cardioembolic ischemic stroke occurred in $5.3 \%$ of patients on colchicine versus $16 \%$ of patients on the placebo arm (HR: $0.33 ; 95 \% \mathrm{Cl}, 0.18$ to $0.59 ; \mathrm{p}<0.001)$. Therefore, the LoDoCo trial provided evidence suggesting that colchicine may be beneficialinpreventing recurrent cardiovascular events in patients with stable angina.

In light of these encouraging preliminary results, the Colchicine Cardiovascular Outcomes Trial (COLCOT) was designed to examine the effects of low dose colchicine on cardiovascular events in over 4500 patients with an acute coronary syndrome. ${ }^{39}$ In the COLCOT trial, patients were randomized within 30 days of an acute coronary syndrome and after planned percutaneous revascularization to the standard of care and colchicine $0.5 \mathrm{mg} /$ day or placebo. The primary efficacy end point was a composite of death from cardiovascular causes, resuscitated cardiac arrest, myocardial infarction, stroke, or urgent hospitalization for angina leading to coronary revascularization.Patients were followed for a median of 22.6 months. The primary end point occurred in $5.5 \%$ of the patients in the colchicine group versus $7.1 \%$ of those in the placebo arm (HR: 0.77 ; $95 \% \mathrm{Cl}: 0.61$ to $0.96 ; \mathrm{p}=0.02)$. The HR were $0.84(95 \% \mathrm{Cl}$, 0.46 to 1.52 ) for death from cardiovascular causes, 0.83 (95\% Cl, 0.25 to 2.73 ) for resuscitated cardiac arrest, 0.91 (95\% Cl, 0.68 to 1.21 ) for myocardial infarction, 0.26 (95\% $\mathrm{Cl}, 0.10$ to 0.70$)$ for stroke, and $0.50(95 \% \mathrm{Cl}, 0.31$ to 0.81$)$ for urgent hospitalization for angina leading to coronary revascularization. Considering that a) postinfarction pericarditis typically occurs within the first few days post-MI and that b) the mean time from the index myocardial infarction to randomization was 13.5 days, it is unlikely that the therapeutic effect of colchicine on pericarditis influenced COLCOT outcomes.

It should also be emphasized that the benefits of colchicine with regard to cardiovascular end points in COLCOT were at least as large as those of canakinumab in CANTOS. ${ }^{33}$ In contrast to canakinumab, however, colchicine did not increase the incidence of septic shock in the COLCOT trial.

\section{NLRP3 inhibitors}

MCC950 and other small-molecule inhibitors of NLRP3, have been developed to treat a variety of inflammasomedriven diseases includingCAD. MCC950 was recently shown to reverse the accelerated atherosclerosis phenotype in mice with myeloid TET2 deficiency ${ }^{17}$ and may be particularly beneficial inCHIP-associated atherosclerotic disease. A trial to assess MCC950 and more selective small-molecule compounds in patients with ischemic heart disease is currently under way. NLRP3 inhibition will also lead to a reduction of active IL-18 in additioncurtailing IL-1 1 p production, which is expected to provide extra protection against atherosclerotic disease. ${ }^{40}$ The most important side-effect of NLRP3 inhibitors is the increased risk of infections. 


\section{Immunomodulators with little to no benefit in atheromatosis}

Cardiovascular risk is increased in patients with autoimmune and rheumatologic disorders. Methotrexate, in addition to curtailing the secretion of pro-inflammatory cytokines, also influences cholesterol transport. ${ }^{41}$ Particularly, in-vitro studies have found that methotrexate inhibits the degradation of the reverse cholesterol transport proteins 27-hydroxylase and ATP-binding cassette transporter A1 (ABCA1) via activation of the $A 2 A$ adenosine receptor. (41) Immunomodulators also exert their anti-atherogenic effects via reducing the expression of vascular adhesion molecules such as VCAM- 1 and ICAM-1, which enhances overall endothelial function. ${ }^{42}$

To test methotrexate in CAD, the phase 3 Cardiovascular Inflammation ReductionTrial (CIRT), recruited 4,786 patients with previous MI or multivessel CAD who additionally had either type 2 diabetes or metabolic syndrome and randomized them to either low-dose methotrexate or placebo..$^{43}$ The trial was stopped after two years due to a lack of difference between groups in the primary composite endpoint of MI, stroke, cardiovascular death, or unstable angina. A reason for the negative result is perhaps the inability of lowdose methotrexate to reduce inflammation (no effect on hsCRP, IL-1b, or IL-6) in patients with CAD, particularly when median hsCRP levels at baseline $(1.6 \mathrm{mg} / \mathrm{l})$ are substantially lower in comparison with patients who present with flares of rheumatoid or psoriatic arthritis.
Multiple additional pathways have been proposed as potential targets for the prevention and treatment of cardiovascular diseases. No benefit has been proven from targeting oxidized LDL (succinobulol) ${ }^{44}$, secretory phospholipase A2 (Verespladib) ${ }^{45}$, lipoprotein-associated phospholipase A2 (Darapladib) ${ }^{46}$, P-selectin (Inclacumab) ${ }^{47}$, IL-1RI (Anakinra) ${ }^{48}$, IL-1A (Xilonix) ${ }^{49}$, or 5-lipoxygenase (Atreleuton) ${ }^{50}$, p38 mitogen-activated protein kinase (Losmapimod). ${ }^{51}$

\section{Conclusions}

Atheromatosis is primarily an inflammatory process. Interestingly, many of the seminal medications used in cardiovascular patients have off-target anti-inflammatory properties. Several immunomodulators are also being investigated as adjuncts to standard of care treatment for atheromatosis. Importantly, the CANTOS trial found that canakinumab, an IL-1b monoclonal antibody, significantly reduces major cardiovascular events while increasing the risk of sepsis. The LoDoCo and the subsequent COLCOT trial, showed that low-dose colchicine also has affords significant cardiovascular protection without predisposing to infections. To date, no benefit has been shown from targeting other aspects of the inflammatory cascade.

\section{Acknowledgements}

Dr. Mylonas is supported with a grant by the Hellenic Atherosclerosis Society for his PhD research.

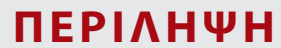

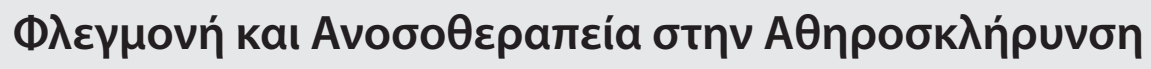

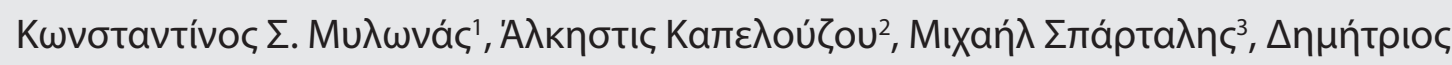

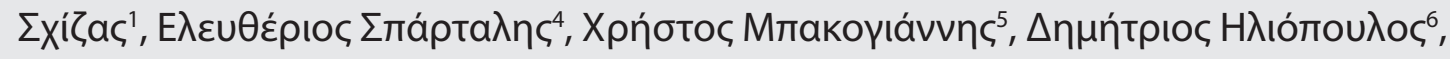

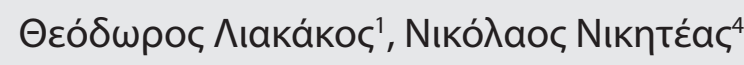

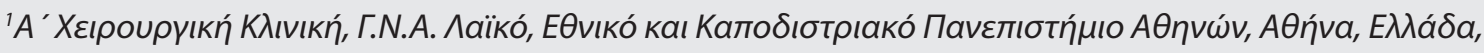

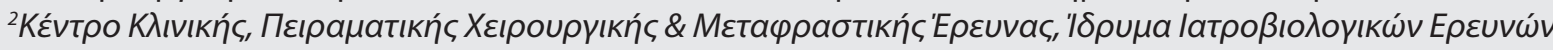

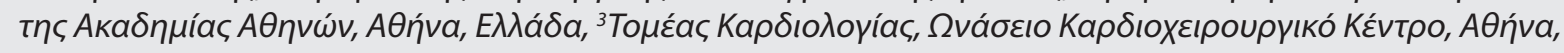

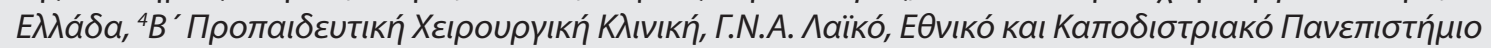

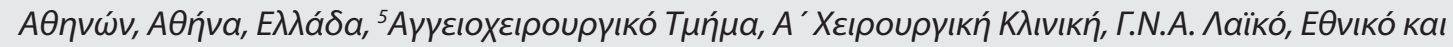

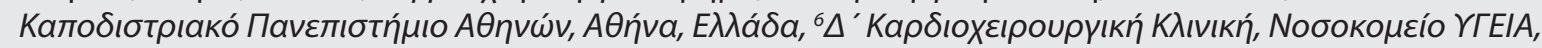

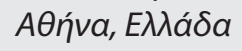

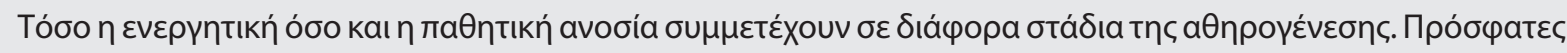

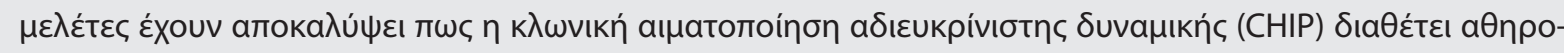

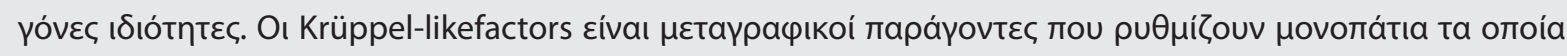

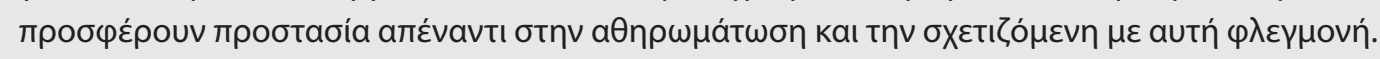




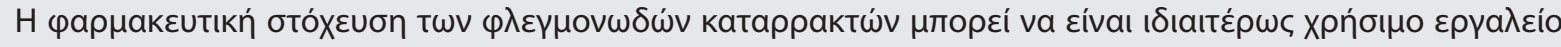

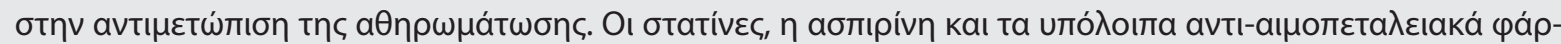

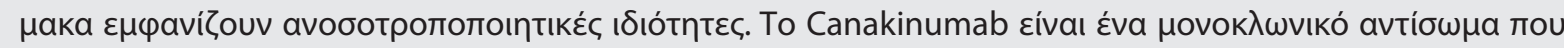

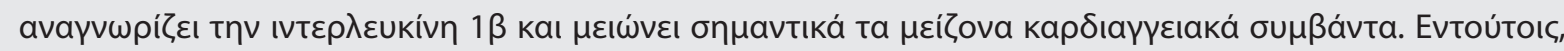

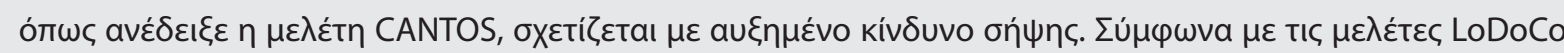

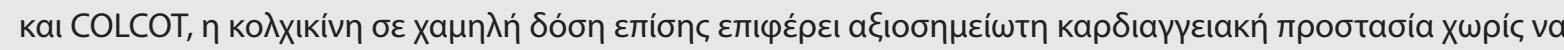

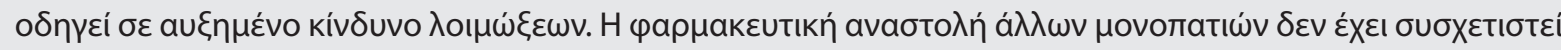

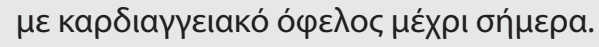

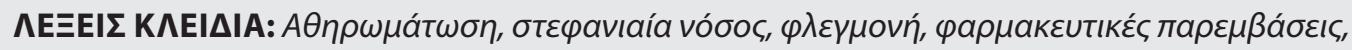

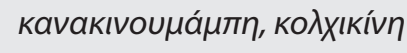

\section{REFERENCES}

1. Davies PF. Endothelial transcriptome profiles in vivo in complex arterial flow fields. Ann Biomed Eng. 2008 Apr;36(4):563-70.

2. Papoutsis K, Kapelouzou A, Georgiopoulos G, Kontogiannis C, Kourek C, Mylonas KS, et al. Tissue-specific relaxin-2 is differentially associated with the presence/size of an arterial aneurysm and the severity of atherosclerotic disease in humans. Acta Pharmacol Sin [Internet]. 2020 Feb. Available from: https://www.nature.com/articles/ s41401-019-0350-5

3. Davies PF. Hemodynamic shear stress and the endothelium in cardiovascular pathophysiology. Nat Clin Pract Cardiovasc Med. 2009 Jan;6(1):16-26.

4. Packard RR, Libby P. Inflammation in atherosclerosis: from vascular biology to biomarker discovery and risk prediction. Clin Chem. 2008 Jan;54(1):24-38.

5. Weber C, Noels H. Atherosclerosis: current pathogenesis and therapeutic options. Nat Med. 2011 Nov; 17(11):141022.

6. Libby P, Ridker PM, Hansson GK. Progress and challenges in translating the biology of atherosclerosis. Nature. 2011 May;473(7347):317-25.

7. Stewart CR, Stuart LM, Wilkinson K, van Gils JM, Deng J, Halle A, et al. CD36 ligands promote sterile inflammation through assembly of a Toll-like receptor 4 and 6 heterodimer. Nat Immunol. 2010 Feb;11(2):155-61.

8. Duewell P, Kono H, Rayner KJ, Sirois CM, Vladimer G, Bauernfeind FG, et al. NLRP3 inflammasomes are required for atherogenesis and activated by cholesterol crystals. Nature. 2010 Apr;464(7293):1357-61.

9. Sheedy FJ, Moore KJ. IL-1 signaling in atherosclerosis: sibling rivalry. Nat Immunol. 2013 Sep ;14(10):1030-2.

10. Zheng Y, Gardner SE, Clarke MC. Cell death, damageassociated molecular patterns, and sterile inflammation in cardiovascular disease. Arterioscler Thromb Vasc Biol. 2011 Dec;31(12):2781-6.

11. Gistera A, Robertson AK, Andersson J, Ketelhuth DF, Ovchinnikova O, Nilsson SK, et al. Transforming growth factor-beta signaling in T cells promotes stabilization of atherosclerotic plaques through an interleukin-17-dependent pathway. Sci Transl Med. 2013 Jul;5(196):196ra100.
12. Gomez D, Owens GK. Smooth muscle cell phenotypic switching in atherosclerosis. Cardiovasc Res. 2012 Jul;95(2):156-64.

13. Jaiswal S, Fontanillas P, Flannick J, Manning A, Grauman PV, Mar BG, et al. Age-related clonal hematopoiesis associated with adverse outcomes. N Engl J Med. 2014 Dec;371(26):2488-98

14. Jaiswal S, Natarajan P, Silver AJ, Gibson CJ, Bick AG, Shvartz E, et al. Clonal Hematopoiesis and Risk of Atherosclerotic Cardiovascular Disease. N Engl J Med. 2017 Jul; 377(2): 111-121.

15. Hasselbalch HC. Perspectives on chronic inflammation in essential thrombocythemia, polycythemia vera, and myelofibrosis: is chronic inflammation a trigger and driver of clonal evolution and development of accelerated atherosclerosis and second cancer? Blood. 2012 Apr;119(14):3219-25.

16. Bonnefond A, Skrobek B, Lobbens S, Eury E, Thuillier D, Cauchi $S$, et al. Association between large detectable clonal mosaicism and type 2 diabetes with vascular complications. Nat Genet. 2013 Sep;45(9):1040-3.

17. Fuster JJ, MacLauchlan S, Zuriaga MA, Polackal MN, Ostriker AC, Chakraborty R, et al. Clonal hematopoiesis associated with TET2 deficiency accelerates atherosclerosis development in mice. Science (New York, NY). 2017 Feb;355(6327):842-7.

18. Cull AH, Snetsinger B, Buckstein R, Wells RA, Rauh MJ. Tet2 restrains inflammatory gene expression in macrophages. Exp Hematol. 2017 Nov;55:56-70.e13.

19. Hamik A, Lin Z, Kumar A, Balcells M, Sinha S, Katz J, et al. Kruppel-like factor 4 regulates endothelial inflammation. J Biol Chem. 2007 May;282(18):13769-79.

20. Li Z, Martin M, Zhang J, Huang HY, Bai L, Zhang J, et al. Kruppel-Like Factor 4 Regulation of Cholesterol-25-Hydroxylase and Liver X Receptor Mitigates Atherosclerosis Susceptibility. Circulation. 2017 Oct;136(14):1315-30.

21. Zhou G, Hamik A, Nayak L, Tian H, Shi H, Lu Y, et al. Endothelial Kruppel-like factor 4 protects against atherothrombosis in mice. J Clin Invest. 2012 Dec;122(12):472731.

22. He M, Huang TS, Li S, Hong HC, Chen Z, Martin M, et al. Atheroprotective Flow Upregulates ITPR3 (Inositol 1,4,5-Trisphosphate Receptor 3) in Vascular Endothe- 
lium via KLF4 (Kruppel-Like Factor 4)-Mediated Histone Modifications. Arterioscler Thromb Vasc Biol. 2019 May;39(5):902-14.

23. Cowan CE, Kohler EE, Dugan TA, Mirza MK, Malik AB, Wary KK. Kruppel-like factor-4 transcriptionally regulates VEcadherin expression and endothelial barrier function. Circ Res. 2010 Oct;107(8):959-66.

24. Denis JF, Diagbouga MR, Molica F, Hautefort A, LinnerzT, Watanabe M, et al. KLF4-Induced Connexin40 Expression Contributes to Arterial Endothelial Quiescence. Front Physiol. 2019 Feb;10:80

25. Liao X, Sharma N, Kapadia F, Zhou G, Lu Y, Hong H, et al. Kruppel-like factor 4 regulates macrophage polarization. J Clin Invest. 2011 Jul;121(7):2736-49.

26. Jain MK, Ridker PM. Anti-inflammatory effects of statins: clinical evidence and basic mechanisms. Nat Rev Drug Discov. 2005 Dec;4(12):977-87.

27. Ii M, Losordo DW. Statins and the endothelium. Vascul Pharmacol. 2007 Jan;46(1):1-9.

28. Paul-Clark MJ, Van Cao T, Moradi-Bidhendi N, Cooper D, Gilroy DW. 15-epi-lipoxin A4-mediated induction of nitric oxide explains how aspirin inhibits acute inflammation. J Exp Med. 2004 Jul 5; 200(1): 69-78.

29. De Haro J, Bleda S, Laime IV, Carballido B, Uyaguari J, Acin F. Aspirin-Dependent Platelet Inflammatory Inhibition in Healthy Subjects Decreases NLRP-1 Inflammasome. Ann Vasc Surg. 2019 Aug;59:244-7.

30. Ridker PM, Cushman M, Stampfer MJ, Tracy RP, Hennekens $\mathrm{CH}$. Inflammation, aspirin, and the risk of cardiovascular disease in apparently healthy men. N Engl J Med. 1997 Apr;336(14):973-9.

31. Thomas MR, Storey RF. Effect of P2Y12 inhibitors on inflammation and immunity. Thromb Haemost. 2015 Aug;114(3):490-7.

32. Wallentin L, Becker RC, Budaj A, Cannon CP, Emanuelsson $\mathrm{H}$, Held C, et al. Ticagrelor versus clopidogrel in patients with acute coronary syndromes. N Engl J Med. 2009 Sep;361(11):1045-57.

33. Ridker PM, Everett BM, Thuren T, MacFadyen JG, Chang WH, Ballantyne $C$, et al. Antiinflammatory Therapy with Canakinumab for Atherosclerotic Disease. N Engl J Med. 2017 Sep;377(12):1119-31.

34. Ridker PM, MacFadyen JG, Everett BM, Libby P, Thuren T, Glynn RJ. Relationship of C-reactive protein reduction to cardiovascular event reduction following treatment with canakinumab: a secondary analysis from the CANTOS randomised controlled trial. Lancet (London, England). 2018 Jan;391(10118):319-28.

35. Ridker PM, MacFadyen JG, Glynn RJ, Koenig W, Libby P, Everett BM, et al. Inhibition of Interleukin-1 beta by Canakinumab and Cardiovascular Outcomes in Patients With Chronic Kidney Disease. J Am Coll Cardiol. 2018 May;71(21):2405-14.

36. Ravelli RB, Gigant B, Curmi PA, Jourdain I, Lachkar S, Sobel $A$, et al. Insight into tubulin regulation from a complex with colchicine and a stathmin-like domain. Nature. 2004 Mar;428(6979):198-202.

37. Perico N, Ostermann D, Bontempeill M, Morigi M, Amuchastegui CS, Zoja C, et al. Colchicine interferes with L-selectin and leukocyte function-associated antigen-1 expression on human $\mathrm{T}$ lymphocytes and inhibits $\mathrm{T}$ cell activation. J Am Soc Nephrol. 1996 Apr;7(4):594-601.

38. Nidorf SM, Eikelboom JW, Budgeon CA, Thompson PL. Low-dose colchicine for secondary prevention of cardiovascular disease. J Am Coll Cardiol. 2013 Jan;61 (4):404-10.

39. Tardif JC, Kouz S, Waters DD, Bertrand OF, Diaz R, Maggioni AP, et al. Efficacy and Safety of Low-Dose Colchicine after Myocardial Infarction. N Engl J Med. 2019 Dec;381(26):2497-505.

40. Mallat Z, Corbaz A, Scoazec A, Besnard S, Leseche G, Chvatchko Y, et al. Expression of interleukin-18 in human atherosclerotic plaques and relation to plaque instability. Circulation. 2001 Oct;104(14):1598-603.

41. Reiss AB, Carsons SE, Anwar K, Rao S, Edelman SD, Zhang $\mathrm{H}$, et al. Atheroprotective effects of methotrexate on reverse cholesterol transport proteins and foam cell transformation in human THP-1 monocyte/macrophages. Arthritis Rheum. 2008 Dec;58(12):3675-83.

42. Gonzalez-Gay MA, Garcia-Unzueta MT, De Matias JM, Gonzalez-Juanatey C, Garcia-Porrua C, Sanchez-Andrade $A$, et al. Influence of anti-TNF-alpha infliximab therapy on adhesion molecules associated with atherogenesis in patients with rheumatoid arthritis. Clin Exp Rheumatol. 2006 Jul-Aug;24(4):373-9.

43. Ridker PM, Everett BM, Pradhan A, MacFadyen JG, Solomon DH, Zaharris E, et al. Low-Dose Methotrexate for the Prevention of Atherosclerotic Events. N Engl J Med. 2019 Feb;380(8):752-62.

44. Tardif JC, McMurray JJ, Klug E, Small R, Schumi J, Choi J, et al. Effects of succinobucol (AGI-1067) after an acute coronary syndrome: a randomised, double-blind, placebo-controlled trial. Lancet (London, England). 2008 May;371(9626):1761-8.

45. Nicholls SJ, Kastelein JJ, Schwartz GG, Bash D, Rosenson RS, Cavender MA, et al. Varespladib and cardiovascular events in patients with an acute coronary syndrome: the VISTA-16 randomized clinical trial. JAMA. 2014 Jan;311(3):252-62.

46. O'Donoghue ML, Braunwald E, White HD, Lukas MA, Tarka E, Steg PG, et al. Effect of darapladib on major coronary events after an acute coronary syndrome: the SOLID-TIMI 52 randomized clinical trial. JAMA. 2014 Sep;312(10):1006-15.

47. Tardif JC, Tanguay JF, Wright SR, Duchatelle V, Petroni $\mathrm{T}$, Gregoire JC, et al. Effects of the P-selectin antagonist inclacumab on myocardial damage after percutaneous coronary intervention for non-ST-segment elevation myocardial infarction: results of the SELECT-ACS trial. J Am Coll Cardiol. 2013 May;61(20):2048-55.

48. Abbate A, Van Tassell BW, Biondi-Zoccai G, Kontos MC, Grizzard JD, Spillman DW, et al. Effects of interleukin-1 blockade with anakinra on adverse cardiac remodeling and heart failure after acute myocardial infarction [from the Virginia Commonwealth University-Anakinra Remodeling Trial (2) (VCU-ART2) pilot study]. Am J Cardiol. 2013 May;111(10):1394-400.

49. El Sayed H, Kerensky R, Stecher M, Mohanty P, Davies $M$. A randomized phase II study of Xilonix, a targeted therapy against interleukin 1alpha, for the prevention of superficial femoral artery restenosis after percutaneous revascularization. J Vasc Surg. 2016 Jan;63(1):133-41.e1.

50. Gaztanaga J, Farkouh M, Rudd JH, BrotzTM, Rosenbaum 
Inflammation in atherosclerosis

D, Mani V, et al. A phase 2 randomized, double-blind, placebo-controlled study of the effect of VIA-2291, a 5-lipoxygenase inhibitor, on vascular inflammation in patients after an acute coronary syndrome. Atherosclerosis. 2015 May;240(1):53-60.
51. O'Donoghue ML, Glaser R, Cavender MA, Aylward PE, Bonaca MP, Budaj A, et al. Effect of Losmapimod on Cardiovascular Outcomes in Patients Hospitalized With Acute Myocardial Infarction: A Randomized Clinical Trial. JAMA. 2016 Apr;315(15):1591-9. 